\title{
Progression in Physical Frailty in Peritoneal Dialysis Patients
}

\author{
Gordon Chun-Kau Chan Jack Kit-Chung Ng Kai-Ming Chow \\ Vickie Wai-Ki Kwong Wing-Fai Pang Phyllis Mei-Shan Cheng Man-Ching Law \\ Chi Bon Leung Philip Kam-Tao Li Cheuk-Chun Szeto
}

Carol \& Richard Yu Peritoneal Dialysis Research Centre, Department of Medicine \& Therapeutics, The Chinese

University of Hong Kong, Hong Kong, China

\section{Keywords}

Renal failure · Peritoneal dialysis · Frailty transitions

\begin{abstract}
Background: Physical frailty contributes to adverse clinical outcomes in peritoneal dialysis (PD) patients. Little has been reported about frailty transitions in this population. We aimed to describe the transitions of frailty in PD patients and identify factors that predicted changes in frailty state. Methods: In a prospective observational study, we recruited 267 PD patients. Frailty was assessed by a validated frailty score. Depression was graded by PHQ-9 score, and nutritional status was evaluated by serum albumin, Subjective Global Assessment (SGA), and comprehensive Malnutrition Inflammation Score (MIS). The primary outcome was the change in frailty score at follow-up compared to baseline. Results: At baseline, 194 (72.7\%) patients were classified as frail. With time, their frailty scores significantly increased $(p<0.001)$, and 93 of the surviving subjects (78.2\%) were classified as frail. There was a modest significant correlation between change in MIS $(p<0.001)$, change in SGA score $(p<0.001)$, and change in PHQ-9 score $(p<0.001)$ with change in frailty score. An increase in PHQ-9 score $(p<0.001)$ and MIS ( $p=$
\end{abstract}

karger@karger.com www.karger.com/kbr

Karger!"
(C) 2021 The Author(s)

Published by S. Karger AG, Basel

This is an Open Access article licensed under the Creative Commons Attribution-NonCommercial-4.0 International License (CC BY-NC) (http://www.karger.com/Services/OpenAccessLicense), applicable to the online version of the article only. Usage and distribution for commercial purposes requires written permission.
0.001 ), as well as longer duration of hospitalization ( $p=$ 0.001 ), was independently associated with a greater change in frailty score after adjustment for confounding factors. Frailty score was also improved in patients who were converted to hemodialysis ( $p=0.048)$ and received renal transplantation ( $p=0.005$ ). Conclusion: Our findings suggested that frailty transitions were common in PD patients. Worsening in nutrition and depression, together with a longer duration of hospitalization, were associated with worsening in frailty.

(C) 2021 The Author(s)

Published by S. Karger AG, Basel

\section{Introduction}

Frailty refers to a state of vulnerability to stressors, and it is typically characterized by weight loss, exhaustion, weakness, slow walking speed, and low physical activity [1]. Frailty causes progressive decline in physical function, thus is regarded as one of the main contributing factors of adverse clinical outcomes including a higher utilization of healthcare resources [2, 3], an increased hospitalization rate [4-6], and a higher mortality rate [4, 5]. 
Frailty is common in the dialysis population $[4,6]$. Impaired renal function causes disturbance in homeostasis, accumulation of uremic toxins, and other metabolic derangements. In addition, anorexia in CKD contributes to the development of malnutrition and frailty [7]. Physical frailty is regarded as a dynamic process as the disease severity changes with age and time [8], especially in dialysis patients as dialysis removes uremic toxins, restores homeostasis, reverses the above detrimental effects, and thus can theoretically improve frailty. Understanding frailty transitions in dialysis patients helps understand the natural course of the disease and to identify high-risk groups and the predictive factors so that we can intervene early to slow down or even reverse the progression. A previous study showed that frailty is prevalent among Chinese peritoneal dialysis (PD) patients $[6,9]$. Frail PD patients have a higher risk of hospitalization, and their hospital stay tends to be prolonged [6]. Frailty also has an additive adverse effect with depressive symptoms on the nutritional status and clinical outcomes [9]. However, previous studies only assessed frailty at the baseline. In addition, published evidence that examined frailty transitions only included patients on hemodialysis (HD) $[10,11]$ but not PD patients. The goal of this study is to examine the change in frailty state with PD therapy. We also attempted to identify predictive factors that are associated with frailty transitions.

\section{Methods}

\section{Study Population}

The study was approved by the local clinical research Ethics Committee. All study procedures were in compliance with the Declaration of Helsinki. We recruited 267 prevalent PD patients from our dialysis unit from January 1, 2015 to December 31, 2016. The study size was based on experience with previous reports [4, 6]. Patients with expected survival of less than 3 months, or those who were planned to receive kidney transplantation in 3 months, were excluded.

After written informed consent, clinical and laboratory data were obtained by chart review. Clinical data comprise patients' age, gender, body weight, height, primary diagnosis of renal disease, and concomitant chronic medical illnesses including diabetes mellitus, ischemic heart disease, cerebrovascular accident, peripheral vascular disease, chronic hepatitis B and C infections, chronic lung disease, malignancy, and immunological diseases. Laboratory assessment included serum urea, creatinine, albumin level, dialysis adequacy by total and peritoneal weekly $\mathrm{Kt} / \mathrm{V}$, and residual renal function as represented by the residual glomerular filtration rate. The peritoneal transporter state was characterized by dialysate-toplasma creatinine concentration at 4 th hour ratio and mass transfer area coefficient.

Frailty Transitions in Peritoneal Dialysis
At the start of the study, we measured their frailty, anthropometry, body mass composition, and pulse wave velocity (PWV) as baseline. Comorbidity load assessment by the Charlson Comorbidity Index (CCI), and nutritional assessment by the comprehensive Malnutrition Inflammation Score (MIS) [12] and Subjective Global Assessment (SGA) [13] were also performed at the same time. After a 4-year observational period, we evaluated their frailty, anthropometry, comorbidity load, and nutritional state again by the same assessment protocol. Body mass composition measurement was also performed in some selected patients.

\section{Assessment of Frailty}

A questionnaire validated in Chinese population was used [6]. The questionnaire consists of 30 yes/no questions, which involves subjective assessment of personal health, psychological state, physical state in terms of number of hospital or doctor visits and medication needs to be taken, body weight, need of assistance in different aspects of daily living, and mobility (online suppl. Fig. 1; see www. karger.com/doi/10.1159/000515635 for all online suppl. material). The total score was calculated, and physical frailty was defined as a score of 6 or above [6]. Frail individuals are further classified into mild frail, moderate frail, and severe frail if they got a frailty score of 6-9, 9-12, and more than 12 , respectively [6].

We also calculated the Clinical Frailty Scale [14] at the followup, a 9-point clinician-based multidimensional assessment that classifies patients' degree of fitness. A patient was ranked from level 1 (very fit) to level 9 (terminally ill) based on case notes review and nursing assessment record retrospectively.

\section{Anthropometric and Body Composition Assessment}

Anthropometric data were measured at recruitment and at the follow-up. General adiposity was measured by BMI. Waist-to-hip ratio, as defined by the ratio between waist circumference and hip circumference, was used to assess central adiposity.

We used the multifrequency bioimpedance spectroscopy device (Body Composition Monitor, Fresenius Medical Care, Germany), which was validated in healthy subjects and dialysis patients [15-17]. In brief, electrodes were attached to one hand and one foot, with the patient in a supine position. The following parameters were computed: volume of overhydration, the ratio of extracellular water to intracellular water, lean tissue mass, and adipose tissue mass.

\section{Pulse Wave Velocity}

PWV was measured by the Vicorder device (SMT Medical $\mathrm{GmbH} \& \mathrm{Co}$.), as described previously [18]. The results were analyzed by the Complior Analyse program (Artech Medical, Pantin, France). Pressure-sensitive transducers were placed over the neck (carotid artery), wrist (radial artery), and groin (femoral artery), with the patient in the supine position. PWV of the carotid-femoral and carotid-radial territories were calculated by dividing the distance between the sensors by the time corresponding to the period separating the start of the rising phase of the carotid pulse wave and that of the femoral and also the radial pulse waves.

\section{Outcome Measures}

All the patients were followed up till December 31, 2019. The primary outcome measure was the change in frailty score during the study period. Besides, we categorized frailty transitions to 4 classes: remained same frail severity, worsening frailty (which refers to a 
Fig. 1. Change in frailty severity from baseline to follow-up assessment. Key: white, remained robust; dark grey, same frailty severity; light grey, improving frailty; black, worsening frailty.

\begin{tabular}{|c|c|c|c|c|c|c|}
\hline \multirow{2}{*}{\multicolumn{2}{|c|}{$\begin{array}{l}\text { No. of cases remained on PD } \\
\text { (No. of cases in whole cohort) }\end{array}$}} & \multicolumn{4}{|c|}{ Follow up assessment } & \multirow{2}{*}{$\begin{array}{r}\text { Total } \\
30\end{array}$} \\
\hline & & \multirow{2}{*}{$\begin{array}{c}\text { None } \\
9 \\
(15)\end{array}$} & \multirow{2}{*}{$\begin{array}{l}\text { Mild frail } \\
\begin{array}{c}8 \\
\text { (9) }\end{array}\end{array}$} & \multirow{2}{*}{$\begin{array}{c}\begin{array}{c}\text { Moderate } \\
\text { frail }\end{array} \\
2 \\
(3)\end{array}$} & \multirow{2}{*}{$\begin{array}{c}\begin{array}{c}\text { Severe } \\
\text { frail }\end{array} \\
11 \\
(12)\end{array}$} & \\
\hline $\begin{array}{l}\text { Baseline } \\
\text { assessment }\end{array}$ & None & & & & & $\begin{array}{l}30 \\
(39)\end{array}$ \\
\hline & Mild frail & $\begin{array}{l}6 \\
(9)\end{array}$ & $\begin{array}{l}4 \\
(5)\end{array}$ & $\begin{array}{c}4 \\
(7)\end{array}$ & $\begin{array}{l}11 \\
(12)\end{array}$ & $\begin{array}{l}25 \\
(33)\end{array}$ \\
\hline & Moderate frail & $\begin{array}{l}0 \\
(0)\end{array}$ & $\begin{array}{l}2 \\
(4)\end{array}$ & $\begin{array}{l}5 \\
(9)\end{array}$ & $\begin{array}{l}12 \\
(13)\end{array}$ & $\begin{array}{l}19 \\
(26)\end{array}$ \\
\hline & Severe frail & $\begin{array}{l}0 \\
(1)\end{array}$ & $\begin{array}{l}0 \\
(0)\end{array}$ & $\begin{array}{l}3 \\
\text { (3) }\end{array}$ & $\begin{array}{l}17 \\
(17)\end{array}$ & $\begin{array}{l}20 \\
(21)\end{array}$ \\
\hline & Total & $\begin{array}{l}15 \\
(25)\end{array}$ & $\begin{array}{l}14 \\
(18)\end{array}$ & $\begin{array}{l}14 \\
(22)\end{array}$ & $\begin{array}{l}51 \\
(74)\end{array}$ & $\begin{array}{c}94 \\
(119)\end{array}$ \\
\hline
\end{tabular}

transition of the frailty class from a milder one to a more severe one), improving frailty (which refers to a transition of the frailty class from more severe one to a milder one), and remained robust.

\section{Statistics}

Statistical analysis was performed by SPSS for Windows software version 24 (SPSS Inc., Chicago, IL, USA). Descriptive data were presented as mean \pm SD if normally distributed, or median (interquartile range) otherwise. Clinical parameters were compared by the Student $t$-test, one-way ANOVA, Mann-Whitney U test, or Kruskal-Wallis test for continuous variables, and the $\chi^{2}$ test for categorical variables. $p<0.05$ was considered to be statistically significant in this study. All probabilities were two-tailed.

Multivariate linear regression models were constructed to further identify independent factors associated with change in frailty score after adjustment of potential confounders with $p$ values below 0.1 with univariate analysis. Subjects' demographics, concomitant medical conditions, hospitalization over time, baseline frailty scores, parameters of anthropometry, nutrition, comorbidity load, and depression, both at baseline and at follow-up, together with the absolute change over time, were included to construct the linear regression model as these factors were believed to be closely linked to frailty transitions based on previous studies in the general and elderly population [19-21]. Backward stepwise analysis was used to remove insignificant variables. $p<0.05$ was considered to be statistically significant in this study. All probabilities were two-tailed.

\section{Results}

A total of 267 PD patients were recruited. At baseline, 73 patients $(27.3 \%)$ were classified as robust, $74(27.7 \%)$ mildly frail, 51 (19.1\%) moderately frail, and 69 (25.8\%) severely frail. With $45.7 \pm 6.0$ months of follow-up, 142 adults died, 2 were transferred to another dialysis center, and 4 refused consent. The baseline clinical and biochemical characteristics of the patients who died before following up were summarized and compared in online suppl. Table 1 . The patients who died before following up were more likely to be older diabetic men. They were also more malnourished, frail, and obese. Follow-up assessment was conducted in the remaining 119 subjects at $45.8 \pm 6.04$ months after the first assessment, in which 94 of them remained on PD, 10 were converted to $\mathrm{HD}$, and 15 underwent renal transplantation.

Of the 94 patients who remained on PD, the frailty score increased from $7.83 \pm 4.56$ at baseline to $12.77 \pm$ 7.53 at follow-up $(p<0.001)$. Their CFS scale was $4.03 \pm$ 1.93 at the follow-up, and the frailty score significantly correlated with CFS scale (Pearson correlation coefficient, $r=0.665, p<0.001)$. Among 17 patients who were severe frail, their frailty score increased by $4.71 \pm 6.11$ at the follow-up. Thirteen of them (76.5\%) showed a decline in the frailty score during the follow-up, with a mean change of $7.23 \pm 4.19$ in frailty score. There was a significant transition of frailty group from baseline to follow-up assessment (Wilcoxon matched pairs signed ranks test, $p<0.001)$, as summarized in Figure 1.

\section{Progression in Frailty in PD Cases, and Their Clinical Characteristics}

The clinical characteristics of the subjects who remained on PD on follow-up and their frailty severity at 
Table 1. Demographics and clinical characteristics of patients who remained on PD, according to their change in frailty state

\begin{tabular}{|c|c|c|c|c|c|}
\hline & $\begin{array}{l}\text { Same frailty severity } \\
(n=26)\end{array}$ & $\begin{array}{l}\text { Worsening frailty } \\
(n=47)\end{array}$ & $\begin{array}{l}\text { Improving frailty } \\
(n=11)\end{array}$ & $\begin{array}{l}\text { Remained robust } \\
(n=10)\end{array}$ & $p$ value \\
\hline Age, years & $58.50 \pm 10.55$ & $57.33 \pm 11.39$ & $57.67 \pm 11.20$ & $57.70 \pm 8.95$ & $0.9^{\mathrm{a}}$ \\
\hline \multicolumn{6}{|l|}{ Primary diagnosis, $n(\%)$} \\
\hline $\mathrm{HT}$ & $4(15.4)$ & $8(16.7)$ & $3(27.3)$ & $0(0)$ & \\
\hline $\mathrm{DM}$ & $4(15.4)$ & $10(20.8)$ & $1(9.1)$ & $1(10.0)$ & \\
\hline PKD & $2(7.7)$ & $0(0)$ & $0(0)$ & $1(10.0)$ & $0.1^{\mathrm{b}}$ \\
\hline Urology & $3(11.5)$ & $0(0)$ & $0(0)$ & $0(0)$ & \\
\hline Others & $0(0)$ & $2(4.2)$ & $0(0)$ & $1(10.0)$ & \\
\hline Unknown & $6(23.1)$ & $5(10.4)$ & $1(9.1)$ & $1(10.0)$ & \\
\hline Dialysis duration, months & $54.11 \pm 79.70$ & $44.19 \pm 46.96$ & $23.68 \pm 20.82$ & $49.82 \pm 40.95$ & $0.5^{\mathrm{a}}$ \\
\hline \multicolumn{6}{|l|}{ Peritoneal transport characteristics } \\
\hline \multicolumn{6}{|l|}{$\mathrm{PWV}, \mathrm{cm} / \mathrm{s}$} \\
\hline CF-PWV & $10.66 \pm 2.14$ & $10.88 \pm 1.77$ & $9.18 \pm 1.19$ & $10.16 \pm 1.89$ & $0.3^{\mathrm{a}}$ \\
\hline CR-PWV & $10.46 \pm 1.69$ & $10.44 \pm 1.75$ & $10.77 \pm 1.80$ & $10.10 \pm 0.94$ & $0.9^{\mathrm{a}}$ \\
\hline Dialysis adequacy (timed-average total Kt/V) & $1.69 \pm 0.27$ & $1.87 \pm 0.34$ & $1.96 \pm 0.38$ & $1.94 \pm 0.23$ & $0.04^{\mathrm{a}}$ \\
\hline Hospitalization, $n^{*}$ & $10[3-17.5]$ & $10[5-18.5]$ & $5[4-10]$ & $2[1-4]$ & $0.02^{\mathrm{c}}$ \\
\hline Hospitalization duration, days* & $26[10-61.5]$ & $31[14.5-62]$ & $27[11-72]$ & $5.5[2.5-32]$ & $0.2^{c}$ \\
\hline
\end{tabular}

PD, peritoneal dialysis; DM, diabetes mellitus; GN, glomerulonephritis; PKD, polycystic kidney disease; D/P4, dialysate-to-plasma creatinine concentration at 4th hour; MTAC, mass transfer area coefficient; HT, hypertension; NPNA, normalized protein nitrogen appearance; FEBM, fat-free edema-free body mass; PWV, pulse wave velocity; CF-PWV, carotid-femoral pulse wave velocity; CR-PWV, carotid-radial pulse wave velocity. ${ }^{a}$ Data were compared by one-way ANOVA. ${ }^{b}$ Data were compared by $\chi^{2}$ test. ${ }^{c}$ Data were compared by Kruskal-Wallis test. * Data presented as median [interquartile range].

different assessments are summarized in Tables 1 and 2 , and Figure 1, respectively. Patients who remained robust had a lower mass transfer area coefficient of creatinine than other groups. Patients who remained frail were poorly dialyzed during their course of therapy with the lowest timed average $\mathrm{Kt} / \mathrm{V}$, and they had the worst nutrition with lowest SGA and highest MIS among the 4 groups. We also observed a significant difference in both baseline and follow-up PHQ-9 scores among the 4 groups $(p<0.001$ and $p=0.02$, respectively). In particular, subjects who remained frail had a significantly higher baseline PHQ-9 score than those with improving frailty and those who remained robust (Student's $t$-test, $p<0.001$ and $p=0.001$, respectively). On the other hand, patients who remained robust had a significantly lower follow-up PHQ-9 score than those who remained frail and those with worsening frailty $(p=$ 0.04 and 0.01 , respectively).

Frailty Transitions in Peritoneal Dialysis
During the observation period, the number of hospital admissions was significantly different between the groups (Kruskal-Wallis test, $p=0.02$ ) (Fig. 2a). We also observed a significant difference in the number of hospital admissions between subjects who remained frail and those who remained robust (Mann-Whitney $U$ test, $p=0.002$ ), and subjects with worsening frailty and those who remained robust $(p=0.02)$. Despite a significant difference in the number of hospitalizations, the total duration of hospital stay was similar among 4 groups (Kruskal-Wallis test, $p=$ 0.16) (Fig. 2b).

Further analysis was performed after dividing our cohort into 2 groups: (1) deteriorating frailty (refers to subjects who became more frail, or already severe frail at baseline) and (2) stable or improving frailty (online suppl. Table 3). Patients who belong to the stable or improving frailty group had a significantly better nutritional state at follow-up (higher SGA and lower MIS), despite a comparable baseline nutritional score. 


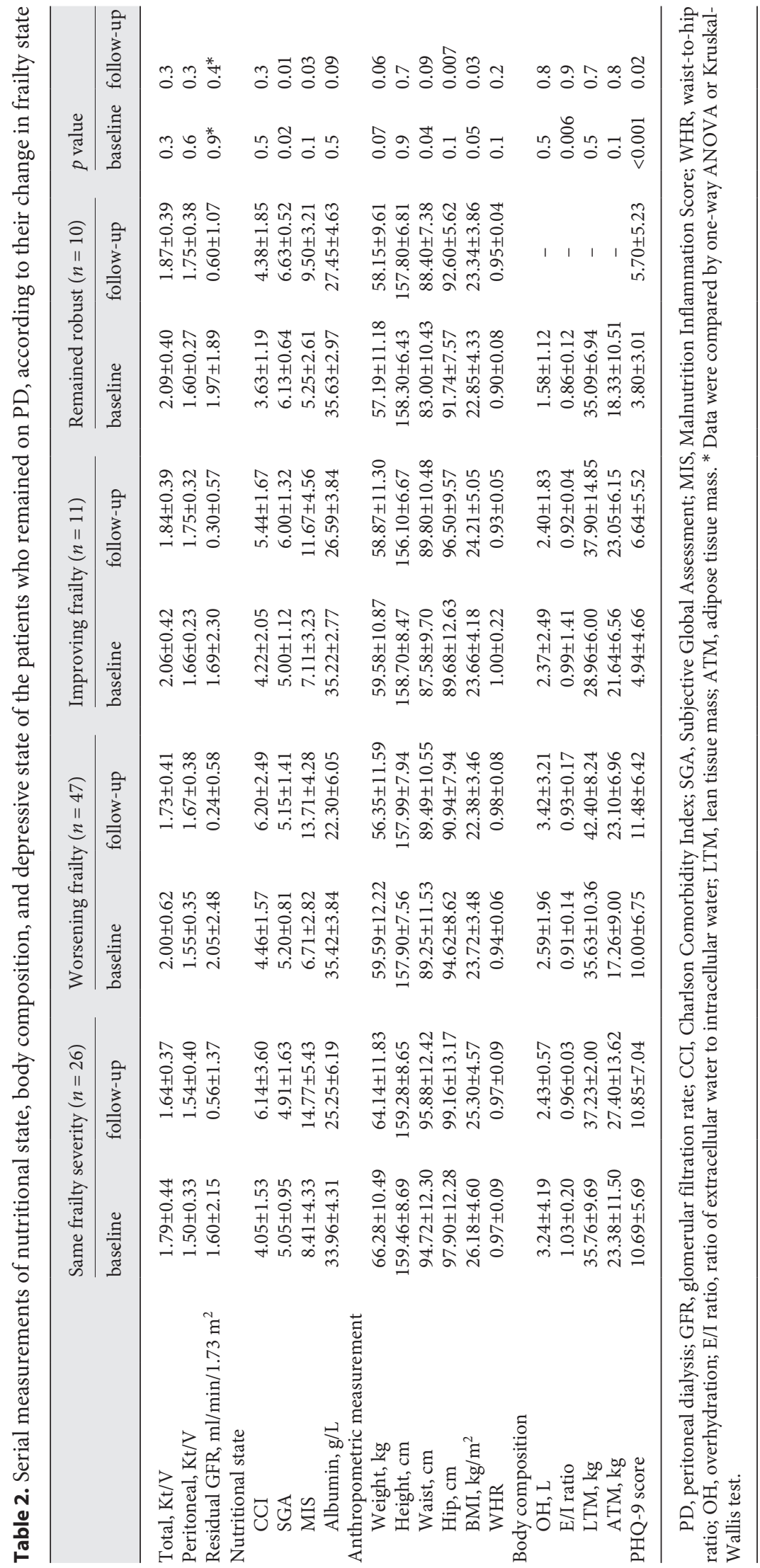




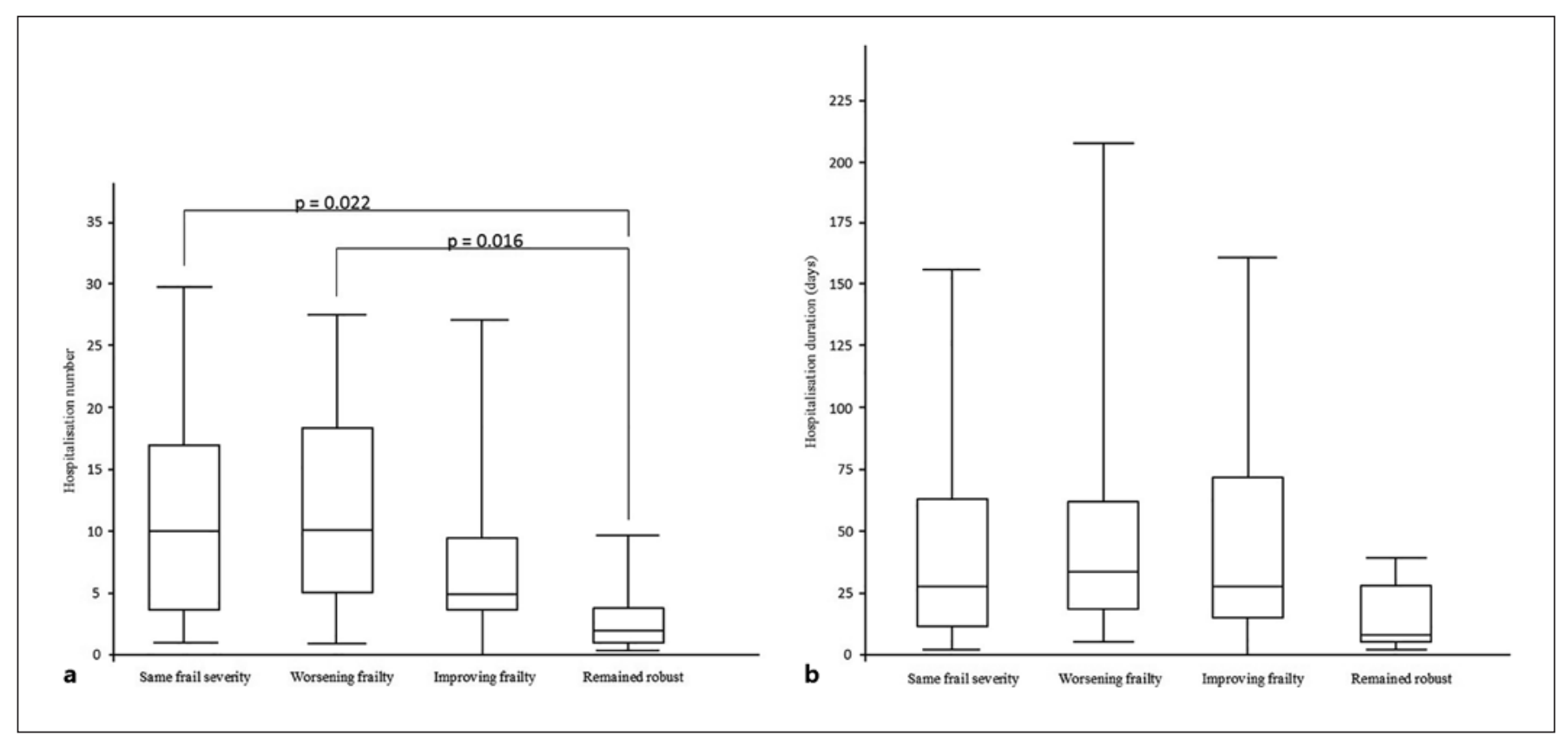

Fig. 2. Need of hospitalization according to the change in frailty state during the study period: number of hospital admissions (a) and duration of hospital stay (b). Whisker box plots, with boxes indicate median, 25th and 75th percentiles, whiskers indicate 5th and 95th percentiles. Data are compared by the Kruskal-Wallis test (a overall $p=0.024$. b overall $p=0.158$ ).

\section{Predictors of Change in Frailty Score}

We analyzed the factors that had correlation with frailty score change, and the result is summarized in Table 3. We found a significant correlation between change in the MIS, SGA score, and depression score (PHQ-9) with frailty score change. (Pearson correlation coefficient, $r=$ $0.437,-0.417$, and 0.569 , respectively, $p<0.001$ for all comparisons). However, there was no significant correlation between frailty score change with other nutritional markers or peritoneal transport characteristics $(r=$ $-0.002,0.040$, and $0.004, p=0.9,0.4$, and 0.9 , respectively, for change in albumin, baseline mass transfer area coefficient, and dialysate-to-plasma creatinine concentration at 4 th hour ratio).

After multivariate adjustment (see Table 3), the duration of hospitalization during the study period, change in MIS, and change in PHQ-9 score were independently associated with the change in frailty score. In this model, each unit increase in the frailty score during the study period was associated with an extra 30.3 days of hospital stay (95\% confidence interval, 19.6-71.4 days).
Impact on Nutritional State, Frailty, and Depression after Conversion to HD or Received Renal

\section{Transplantation}

We also compared the clinical characteristics, nutritional state, frailty, and depression among patients who remained on PD to those who were changed to HD or received kidney transplant. The results are summarized in online suppl. Table 2. In essence, patients who received renal transplantation were younger and less frail at baseline. At the time of the follow-up, transplanted patients had a significantly lower prevalence of frailty ( 57.1 vs. $83.2 \%, p=0.02$ ), a smaller rise in frailty score (0.21 vs. $4.92, p=0.02$ ), and a lower follow-up frailty score ( 6.93 vs. $12.77, p=0.005)$ than patients who remained on PD. Despite a similar baseline depression score, patients who received renal transplantation had an improvement in their PHQ-9 score, whereas those who remained on PD had an increase in PHQ-9 score $(-2.43$ vs. $3.16, p=0.006)$. Patients who were converted to HD were also younger than those who remained on PD. Although they had a similar baseline frailty score, patients converted to HD had a significantly smaller increase in frailty score during follow-up ( 0.30 vs. 4.92 , $p=0.05)$. There was no significant difference in base- 
Table 3. Linear regression analysis on the predictors of frailty score change

\begin{tabular}{|c|c|c|c|c|}
\hline \multirow[t]{2}{*}{ Factor } & \multicolumn{2}{|l|}{ Univariate analysis } & \multicolumn{2}{|l|}{ Multivariate analysis } \\
\hline & $\begin{array}{l}\text { unstandardized B } \\
(95 \% \text { CI })\end{array}$ & $p$ value & $\begin{array}{l}\text { unstandardized B } \\
(95 \% \text { CI })\end{array}$ & $p$ value \\
\hline \multicolumn{5}{|c|}{ Demographics and clinical characteristics } \\
\hline Baseline age, years & $0.121(-0.017$ to 0.259$)$ & 0.08 & & \\
\hline Baseline CF-PWV & $0.814(0.071-1.558)$ & 0.03 & & \\
\hline \multicolumn{5}{|l|}{ Anthropometric measurements } \\
\hline Change in weight & $-0.413(-0.648$ to -0.179$)$ & 0.001 & & \\
\hline Change in hip circumference & $-2.303(-3.800$ to -0.806$)$ & 0.003 & & \\
\hline Change in BMI & $-0.654(-1.167$ to -0.141$)$ & 0.01 & & \\
\hline Change in WHR & $22.373(8.153-36.593)$ & 0.002 & & \\
\hline \multicolumn{5}{|c|}{ Nutritional state and comorbidity load } \\
\hline Baseline frailty score & $-0.374(-0.688$ to -0.060$)$ & 0.02 & & \\
\hline \multicolumn{5}{|l|}{ Depression assessment } \\
\hline Change in PHQ-9 & $0.565(0.397-0.733)$ & $<0.001$ & $0.487(0.316-0.658)$ & $<0.001$ \\
\hline
\end{tabular}

line, follow-up, and change in PHQ-9 score between those who remained on PD and those who were converted to HD.

\section{Discussion}

This is the first study to report frailty transitions among Chinese PD patients. More than half of our cohort had persistent or progression in their frailty after over 3 years of PD. Such transitions were closely related to their body composition, nutrition, and the presence of depressive symptoms. We found that more frequent hospitalization, worsening in depressive symptoms, and nutritional status predicted the progression in physical frailty. Our study is also the first one to examine frailty transitions after switch of dialysis modality to HD or kidney transplantation.

Dialysis therapy itself may also cause a detrimental effect on nutritional state due to a loss of albumin and nutrients during therapy $[22,23]$. Frailty progression has been recognized in patients undergoing HD [10, 11]. To date, little is known about such progression in PD patients. The observation of frailty transitions in HD pa- tients should not be extrapolated to PD patients due to a number of reasons. First, PD patients are more frequently exposed to glucose-containing dialysate, which provides caloric supplementation [24] and may potentially ameliorate malnutrition. On the other hand, chronic exposure to glucose-containing dialysate causes accumulation of fat [25], which can produce a wide range of proinflammatory mediators like adiponectin [26]. These mediators further aggravate chronic inflammation in our PD patients. PD therapy itself also induces protein loss to the dialysate fluid [27]. Our study on frailty transitions in PD patients is able to fill in the gap of knowledge in this field.

Our study highlights poor nutrition and depression may accelerate frailty progression. Physical frailty, depression, and poor nutrition are known to be interlinked with one another. Malnutrition is a major cause of physical frailty. Physical frailty is closely linked to depression [6] and poor nutrition [9]. On the other hand, physical frailty and depression had a synergistic and additive harmful effect on nutrition [9] and mortality [28]. Our study again reinforced the observation that these 3 domains are closely related.

We adopted 3 assessment tools to assess nutrition, which included MIS, SGA score, and serum albumin. Our 
result showed the SGA score and MIS differed between groups of frailty transitions. The degree of change in SGA score and MIS from baseline to follow-up also predicted a change in frailty score in univariate analysis. However, after adjustment of confounding factors, only change in MIS predicted frailty change. Compared to the serum albumin level and SGA score, MIS is a more preferred tool to assess nutrition in PD patients as it provides a more comprehensive evaluation as it involves assessments of different domains including diet intake, patients' symptoms, functional capacity, and clinical evaluation on fat and muscle mass, BMI, and serum parameters. MIS is well validated in Chinese population [29], and the assessment results are less affected by acute illnesses [29, 30]. It correlates well with systemic inflammatory state, including a systemic inflammatory state [22] and frailty state [6].

Our result showed subjects who transited into a more severe frailty state were more frequently hospitalized (Table 1). On the other hand, the multivariate linear regression analysis with adjustment of confounders showed that only the total hospitalization duration but not frequency of hospitalization was associated with a rise in frailty score with time (Table 3 ). We postulate the difference in results could be related by a larger scale with greater numerical values represented by hospitalization duration than hospitalization frequency. As a result, hospitalization duration is probably a more robust measure in predicting frailty score change based on our observation.

In this study, we also assessed frailty transition in patients who were converted to HD or received kidney transplantation. In short, PD patients who underwent kidney transplantation had an overall better performance in terms of improved nutrition, less depression, and less physical frailty than their counterparts who remained on PD. Such results concurred with previous reports $[31,32]$. As for patient's performance after conversion from $\mathrm{PD}$ to $\mathrm{HD}$, it was not reported in the past. Our study revealed that among all parameters, physical frailty is the only domain that was improved after conversion to HD. It can be explained by the difference in the inflammatory state between $\mathrm{PD}$ and HD patients, as reported in previous studies [11, 26, 33, 34]. Whether switching from PD to HD is an option to slow down frailty progression should be verified with a well-designed controlled trial with a larger number of samples, though it will be difficult to be carried out in real-life situations.

Our study has a few strengths: first, we are the first group of investigators reporting frailty transitions in $\mathrm{PD}$ patients. Second, the follow-up period of this study was more than 4 years. This prolonged window of observation enabled a more thorough understanding on the natural history of the disease progression. Third, we identified predictive factors of frailty transitions, which may have important prognostic implications. Target-based therapy may be undertaken to slow down or improve frailty though that needs to be confirmed with a randomized controlled trial in the future. Our study also has certain limitations. First, our study involved assessment of frailty state at 2 time points, in which we assumed a constant relationship between frailty and time. In reality, physical frailty is a dynamic process in which the degree of change may fluctuate with time. Second, we recruited prevalent, rather than incident, PD patients at the clinic. Theoretically, frailty progression may be affected by the duration of time on dialysis, though our result did not reflect such difference (Table 1). Recruitment of cases at the clinic may cause potential selection bias as we could not recruit cases who were hospitalized or too frail to attend the clinic. Third, we performed follow-up assessment at late 2019 when our subjects attended clinic, which makes the follow-up duration slightly different between subjects. We were also only able to perform follow-up assessment on individuals who remained alive during the follow-up, and this would introduce survival bias. Finally, we defined improving and worsening frailty by a change in frailty severity measured by the questionnaire. There is a chance of underestimating the number of patients who had a change in frailty score, especially in those who had a small change in frailty score. They may still be classified as same frailty or remained robust, unless their final score fell into a different frail severity. However, putting these cases into the group of improving/worsening frailty may reduce the power of our analysis as their change in frailty severity is subclinical and did not significantly impact patients' quality of life.

\section{Conclusion}

Our findings suggest that progression of frailty is common in PD patients. Worsening in nutrition and depression, together with frequent hospital admission, predicted deterioration in frailty state. Whether target-based interventions in those predictive factors would be beneficial requires further studies. 


\section{Acknowledgements}

This work was supported by the Chinese University of Hong Kong (CUHK) research accounts (6905134 and 7105912).

\section{Statement of Ethics}

This study was approved by the Joint Chinese University of Hong Kong - New Territories East Cluster Clinical Research Ethics Committee (CREC Ref. No. 2018.587). All study procedures were in compliance with the World Medical Association Declaration of Helsinki. Written informed consent was obtained from all study subjects.

\section{Conflict of Interest Statement}

The authors declare no conflict of interest. The results of this article have not been published previously in whole or in part, except in abstract format.

\section{Funding Sources}

There was no commercial sponsorship for this study. The funder had no role in study design, data collection and analysis, decision to publish, or preparation of the manuscript.

\section{Author Contributions}

Gordon Chun-Kau Chan: conceptualization, methodology, data curation, formal analysis, writing - original draft, and project administration. Jack Kit-Chung Ng: investigation. Kai-Ming Chow: investigation. Vickie Wai-Ki Kwong: investigation. WingFai Pang: investigation. Phyllis Mei-Shan Cheng: investigation. Man-Ching Law: investigation. Chi-Bon Leung: investigation. Philip Kam-Tao Li: supervision. Cheuk-Chun Szeto: conceptualization, methodology, data curation, writing - review and editing, and supervision.

\section{References}

1 Fried LP, Tangen CM, Walston J, Newman $A B$, Hirsch $C$, Gottdiener J, et al. Frailty in older adults: evidence for a phenotype. J Gerontol A Biol Sci Med Sci. 2001;56(3): M146-56.

2 Bock JO, König HH, Brenner H, Haefeli WE, Quinzler R, Matschinger $\mathrm{H}$, et al. Associations of frailty with health care costs: results of the ESTHER cohort study. BMC Health Serv Res. 2016;16(7):128-53.

3 Morley JE, Vellas B, van Kan GA, Anker SD, Bauer JM, Bernabei R, et al. Frailty consensus: a call to action. J Am Med Dir Assoc. 2013 Jun; 14(6):392-7.

4 Johansen KL, Chertow GM, Jin C, Kutner NG. Significance of frailty among dialysis patients. J Am Soc Nephrol. 2007;18(11):29607.

5 Clegg A, Young J, Iliffe S, Rikkert MO, Rockwood K. Frailty in elderly people. Lancet. 2013 Mar;381(9868):752-62

$6 \mathrm{Ng} \mathrm{JK}$, Kwan BC, Chow KM, Cheng PM, Law MC, Pang WF, et al. Frailty in Chinese Peritoneal dialysis patients: prevalence and prognostic significance. Kidney Blood Press Res. 2016;41(6):736-45.

7 Fouque D, Pelletier S, Mafra D, Chauveau P. Nutrition and chronic kidney disease. Kidney Int. 2011 Aug;80(4):348-57.

8 Gill TM, Gahbauer EA, Allore HG, Han L. Transitions between frailty states among community-living older persons. Arch Intern Med. 2006 Feb 27;166(4):418-23.

9 Szeto CC, Chan GC, Ng JK, Chow KM, Kwan BC, Cheng PM, et al. Depression and physical frailty have additive effect on the nutritional status and clinical outcome of Chinese peritoneal dialysis. Kidney Blood Press Res. 2018; 43(3):914-23.
10 Johansen KL, Dalrymple LS, Delgado C, Chertow GM, Segal MR, Chiang J, et al. Factors associated with frailty and its trajectory among patients on hemodialysis. Clin J Am Soc Nephrol. 2017 Jul 7;12(7):1100-8.

11 Johansen KL, Delgado C, Kaysen GA, Chertow GM, Chiang J, Dalrymple LS, et al. Frailty among patients receiving hemodialysis: evolution of components and associations with mortality. J Gerontol A Biol Sci Med Sci. 2019 Feb 15;74(3):380-6.

12 Kalantar-Zadeh K, Kopple JD, Block G, Humphreys $\mathrm{MH}$. A malnutrition-inflammation score is correlated with morbidity and mortality in maintenance hemodialysis patients. Am J Kidney Dis. 2001;38(6):1251-63.

13 Enia G, Sicuso C, Alati G, Zoccali C. Subjective global assessment of nutrition in dialysis patients. Nephrol Dial Transplant. 1993; 8(10):1094-8

14 Rockwood K, Song X, MacKnight C, Bergman H, Hogan DB, McDowell I, et al. A global clinical measure of fitness and frailty in elderly people. CMAJ. 2005 Aug 30;173(5): 489-95.

15 Moissl UM, Wabel P, Chamney PW, Bosaeus I, Levin NW, Bosy-Westphal A, et al. Body fluid volume determination via body composition spectroscopy in health and disease. Physiol Meas. 2006;27(9):921-33.

16 Cooper BA, Aslani A, Ryan M, Zhu FY, Ibels LS, Allen BJ, et al. Comparing different methods of assessing body composition in endstage renal failure. Kidney Int. 2000;58(1): $408-16$.
17 Kwan BC, Szeto CC, Chow KM, Law MC, Cheng MS, Leung CB, et al. Bioimpedance spectroscopy for the detection of fluid overload in Chinese peritoneal dialysis patients. Perit Dial Int. 2014;34(4):409-16.

18 Szeto CC, Kwan BC, Chow KM, Leung CB, Law MC, Li PK. Prognostic value of arterial pulse wave velocity in peritoneal dialysis patients. Am J Nephrol. 2012;35(2):127-33.

19 Lee JS, Auyeung TW, Leung J, Kwok T, Woo $\mathrm{J}$. Transitions in frailty states among community-living older adults and their associated factors. J Am Med Dir Assoc. 2014;15(4):2816.

20 Pollack LR, Litwack-Harrison S, Cawthon PM, Ensrud K, Lane NE, Barrett-Connor E, et al. Patterns and predictors of frailty transitions in older men: the osteoporoic fractures in men study. J Am Geriatr Soc. 2017;6(11): 2473-9.

21 Barry LC, Murphy TE, Gill TM. Depressive symptoms and functional transitions over time in older persons. Am J Geriatr Psychiatry. 2011;19(9):783-91.

22 Western WM, Kopple JD, Krediet RT, Appel $\mathrm{M}$, Mehrotra R. Dietary protein requirements and dialysate protein losses in chronic peritoneal dialysis patients. Perit Dial Intl. 2007;27: 192-5.

23 Yeun JY, Kaysen GA. Acute phase proteins and peritoneal dialysate albumin loss are the main determinants of serum albumin in peritoneal dialysis patients. Am J Kidney Dis. 1997;30(6):923-7.

24 Burkett J. Metabolic consequences of peritoneal dialysis. Semin Dial. 2004;17(6):498504 
25 Pelicans R, Strauss BJ, Polkinghorrne KR, Kerr PG. Longitudinal body composition changes due to dialysis. Clin J Am Soc Nephrol. 2011;6:1668-75.

26 Huang JW, Yen CJ, Chiang HW, Hung KY, Tsai TJ, Wu KD. Adiponectin in peritoneal dialysis patients: a comparison with hemodialysis patients and subjects with normal renal function. Am J Kidney Dis. 2004 Jun;43(6): 1047-55.

27 Western WM, Kopple JD, Krediet RT, Appel M, Mehrotra R. Dietary protein requirements and dialysate protein losses in chronic peritoneal dialysis patients. Perit Dial Intl. 2007;27: 192-5.
28 Sy J, McCulloch CE, Johansen KL. Depressive symptoms, frailty, and mortality among dialysis patients. Hemodial Int. 2019 Apr;23(2): 239-46.

29 Chan JY, Che KI, Lam KM, Chow KM, Chung $\mathrm{KY}$, Li PK, et al. Comprehensive malnutrition inflammation score as a marker of nutritional status in Chinese peritoneal dialysis patients. Nephrology. 2007;12(2):130-4.

30 Afsar B, Sezer S, Ozdemir FN, Celik H, Elsurer R, Haberal M. Malnutrition-inflammation score is a useful tool in peritoneal dialysis patients. Perit Dial Int. 2006;26:705-11.

31 McAdams-DeMarco MA, Isaacs K, Darko L, Salter ML, Gupta N, King EA, et al. Changes in frailty after kidney transplantation. J Am Geriatr Soc. 2015 Oct;63(10):2152-7.
32 Akman B, Ozdemir FN, Sezer S, Miçozkadioglu $\mathrm{H}$, Haberal M. Depression levels before and after renal transplantation. Transplant Proc. 2004 Jan-Feb;36(1):111-3.

33 Snaedal S, Qureshi AR, Lund SH, Germanis G, Hylander B, Heimbürger O, et al. Dialysis modality and nutritional status are associated with variability of inflammatory markers. Nephrol Dial Transplant. 2016;31(8):1320-7.

34 Yong K, Dogra G, Boudville N, Lim W. Increased inflammatory response in association with the initiation of hemodialysis compared with peritoneal dialysis in a prospective study of end-stage kidney disease patients. Perit Dial Int. 2018 Jan-Feb;38(1):18-23. 